\title{
Single-step purification of specific tRNAs by hydrophobic tagging
}

\author{
Ute Kothe, Alena Paleskava, Andrey L. Konevega, Marina V. Rodnina * \\ Institute of Physical Biochemistry, University of Witten/Herdecke, D-58448 Witten, Germany
}

Received 14 March 2006

Available online 11 May 2006

Studies on translation frequently require large amounts of purified individual tRNAs. However, few purified tRNAs are commercially available. Individual tRNAs can be easily produced by in vitro transcription, but the lack of modifications of the tRNA transcripts may influence or impair their function [1,2]. The purification of specific tRNAs from total tRNA is a laborious process that requires several chromatographic steps [3]. Therefore, developing a simple and rapid method for purification of specific tRNA is of great importance. Here we describe a method for purification of individual tRNAs based on selective tagging of the amino group of specifically charged aminoacyl-tRNAs (aa-tRNAs) ${ }^{1}$ with 9-fluorenylmethylsuccinimidylcarbonat (FmocOSu) (Fig. 1), followed by a single chromatographic purification step, using reversedphase HPLC or hydrophobic interaction chromatography. The method is suitable for all tRNAs and allows up to 20 -fold enrichment of a specific tRNA in less than 1 day effective working time. The materials used are readily available at low cost.

We tested the procedure for two different tRNAs from Escherichia coli: $\mathrm{tRNA}^{\mathrm{Ala}}$ and $\mathrm{tRNA}^{\mathrm{Sec}}$ (the latter being tRNA specific for selenocysteine). As starting material, we used total tRNA, which is commercially available or can be prepared by standard procedures [4]. For the purification of tRNA ${ }^{\text {Ala }}$, we used total tRNA from MRE 600 cells that contained approximately $3 \% \mathrm{tRNA}^{\mathrm{Ala}}$ according to charging with $\left[{ }^{14} \mathrm{C}\right]$ alanine in an analytical aminoacylation assay. $\mathrm{TRNA}^{\mathrm{Sec}}$ is a rare tRNA that is hardly detected in total tRNA $(<1 \%)$. To increase the amount of tRNA ${ }^{\mathrm{Sec}}$ in the initial tRNA preparation, we overproduced tRNA ${ }^{\mathrm{Sec}}$

\footnotetext{
* Corresponding author. Fax: +49 2302926117.

E-mail address: rodnina@uni-wh.de (M.V. Rodnina).

${ }^{1}$ Abbreviations used: aa-tRNA, aminoacyl-tRNA; FmocOSu, 9-fluorenylmethylsuccinimidylcarbonat; DTT, dithiothreitol; TCA, trichloroacetic acid; DMSO, dimethyl sulfoxide; Fmoc-aa-tRNA, fluoren-9-ylmethoxycarbonyl-aa-tRNA; EF-Tu, elongation factor Tu.
}

in BL21(DE3) cells [5] and isolated total tRNA from these cells. tRNA ${ }^{\text {Sec }}$ and tRNA ${ }^{\text {Ser }}$ in total tRNA can be aminoacylated with serine by seryl-tRNA synthetase [5], yielding Ser-tRNA $^{\text {Sec }}$ and Ser-tRNA ${ }^{\text {Ser }}$ [6]. (Further conversion of Ser-tRNA ${ }^{\mathrm{Sec}}$ to Sec-tRNA ${ }^{\mathrm{Sec}}$ requires a number of additional factors and is not addressed here.) Analytical aminoacylation of total tRNA enriched in tRNA ${ }^{\mathrm{Sec}}$ with $\left[{ }^{14} \mathrm{C}\right]$ serine indicated the presence of approximately $30 \%$ tRNA $^{\mathrm{Sec} / \mathrm{Ser}}$ in total tRNA. For preparative aminoacylation, $50-100 \mu \mathrm{M}$ total tRNA, 3\% (v/v) S100 fraction as a source of aa-tRNA synthetases [7], $3 \mathrm{mM}$ ATP, $50-80 \mu \mathrm{M}{ }^{14} \mathrm{C}$-labeled amino acid (alanine [MP Biomedicals] or serine [Moravic Biochemicals]), and $0.005 \mathrm{U} / \mu \mathrm{l}$ inorganic pyrophosphatase (Sigma) were incubated for $60 \mathrm{~min}$ at $37^{\circ} \mathrm{C}$ in aminoacylation buffer $(50 \mathrm{mM}$ Hepes [pH 7.5], $30 \mathrm{mM} \mathrm{KCl}, 10 \mathrm{mM} \mathrm{MgCl}$, and $2 \mathrm{mM}$ dithiothreitol [DTT]). For the following purification steps, it is crucial to obtain specific charging by only one given amino acid. To avoid charging of other tRNAs, the preparation of aminoacyl-tRNA synthetase must be absolutely free of endogenous amino acids, and this can be achieved by a dialysis step. Alternatively, purified aminoacyl-tRNA synthetases can be used. The aminoacylation efficiency was controlled in an aliquot of the reaction mixture by trichloroacetic acid (TCA) precipitation, filtration through a nitrocellulose filter (Sartorius), and scintillation counting in Quickszint 361 cocktail (Zinsser Analytic). Potassium acetate ( $\mathrm{pH} 4.5)$ was added to the reaction mixture to a final concentration of $0.3 \mathrm{M}$, followed by extraction with an equal volume of water-saturated phenol to remove proteins. The aa-tRNA was precipitated from the aqueous phase with 2.5 volumes of cold ethanol, and the pellet of aa-tRNA was dissolved in $0.1 \mathrm{M}$ sodium acetate $(\mathrm{pH} 4.5)$ [8].

In principle, individual tRNAs (or aa-tRNAs) can be separated by chromatography on a reversed-phase HPLC column (LiChrospher WP, instruction manual, Merck). However, when large amounts of tRNA are used, the 


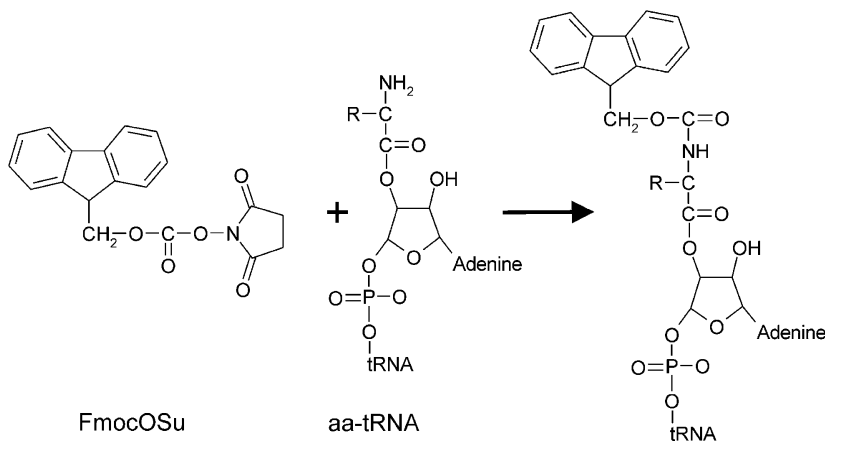

Fig. 1. Formation of fluoren-9-ylmethoxycarbonyl-aa-tRNA (Fmoc-aatRNA). FmocOSu reacts with the free amino group of aa-tRNA. The hydrophobicity of the resulting Fmoc-aa-tRNA is increased significantly compared with deacylated tRNA or unmodified aa-tRNA.

separation of the individual tRNA peaks is quite poor. To change the retention time of a specific aa-tRNA on the column and thereby improve its separation from other (deacylated) tRNAs, we modified the amino group of aa-tRNA with FmocOSu (Sigma-Aldrich), which adds a large aromatic group to the tRNA, thereby increasing its hydrophobicity and retention time on the column (Fig. 1). Succinimide esters selectively modify the amino group of amino acid and do not react with other amino groups in the tRNA molecule [9]. FmocOSu was dissolved in dimethyl sulfoxide (DMSO), 1 volume of tRNA was mixed with 4 volumes of FmocOSu to final concentrations of $20 \mu \mathrm{M}$ aatRNA and $35 \mathrm{mM} \mathrm{FmocOSu}$, and the reaction was carried out for $60 \mathrm{~min}$ at $0{ }^{\circ} \mathrm{C}$, resulting in the formation of fluoren-9-ylmethoxycarbonyl-aa-tRNA (Fmoc-aa-tRNA). Potassium acetate ( $\mathrm{pH} 4.5$ ) was added to $0.3 \mathrm{M}$ final concentration, and Fmoc-aa-tRNA was precipitated with ethanol and dissolved in $0.3 \mathrm{M}$ potassium acetate ( $\mathrm{pH} 4.5)$. To remove unreacted FmocOSu, the sample was phenol-extracted and Fmoc-aa-tRNA was purified by two ethanol precipitations. For the subsequent chromatography, Fmoc-aa-tRNA was dissolved in buffer A $(20 \mathrm{mM}$ ammonium acetate $[\mathrm{pH} 5.0], 10 \mathrm{mM}$ magnesium acetate, and $400 \mathrm{mM} \mathrm{NaCl}$ ).

Separation of Fmoc-aa-tRNA from total tRNA was achieved by chromatography on reversed-phase HPLC using a LiChrospher WP-300 RP-18 $(5 \mu \mathrm{m})$ column $(250 \times 10 \mathrm{~mm})$ (Merck) (Figs. 2A and B). The HPLC system allows the efficient separation of Fmoc-aa-tRNA from deacylated tRNAs and residual unmodified aa-tRNA within $2 \mathrm{~h}$. The tRNA mixture (up to $1700 A_{260}$ units) was applied to the column equilibrated with buffer $\mathrm{A}$, followed by further washing of the column with buffer $\mathrm{A}$ at a flow rate of $3 \mathrm{ml} / \mathrm{min}$. The tRNAs were eluted by a linear gradient from buffer A to $100 \%$ buffer B $(20 \mathrm{mM}$ ammonium acetate $[\mathrm{pH} 5.0], 10 \mathrm{mM}$ magnesium acetate, $400 \mathrm{mM}$ $\mathrm{NaCl}$, and $30 \%[\mathrm{v} / \mathrm{v}]$ ethanol). The elution profile was monitored by measuring absorption $\left(A_{260}\right)$ and scintillation counting of aliquots of eluted fractions to detect Fmoc$\left[{ }^{14} \mathrm{C}\right]$ aa-tRNA. The first small $A_{260}$ peak eluting at $0-15 \%$ buffer B contained traces of ATP from the aminoacylation
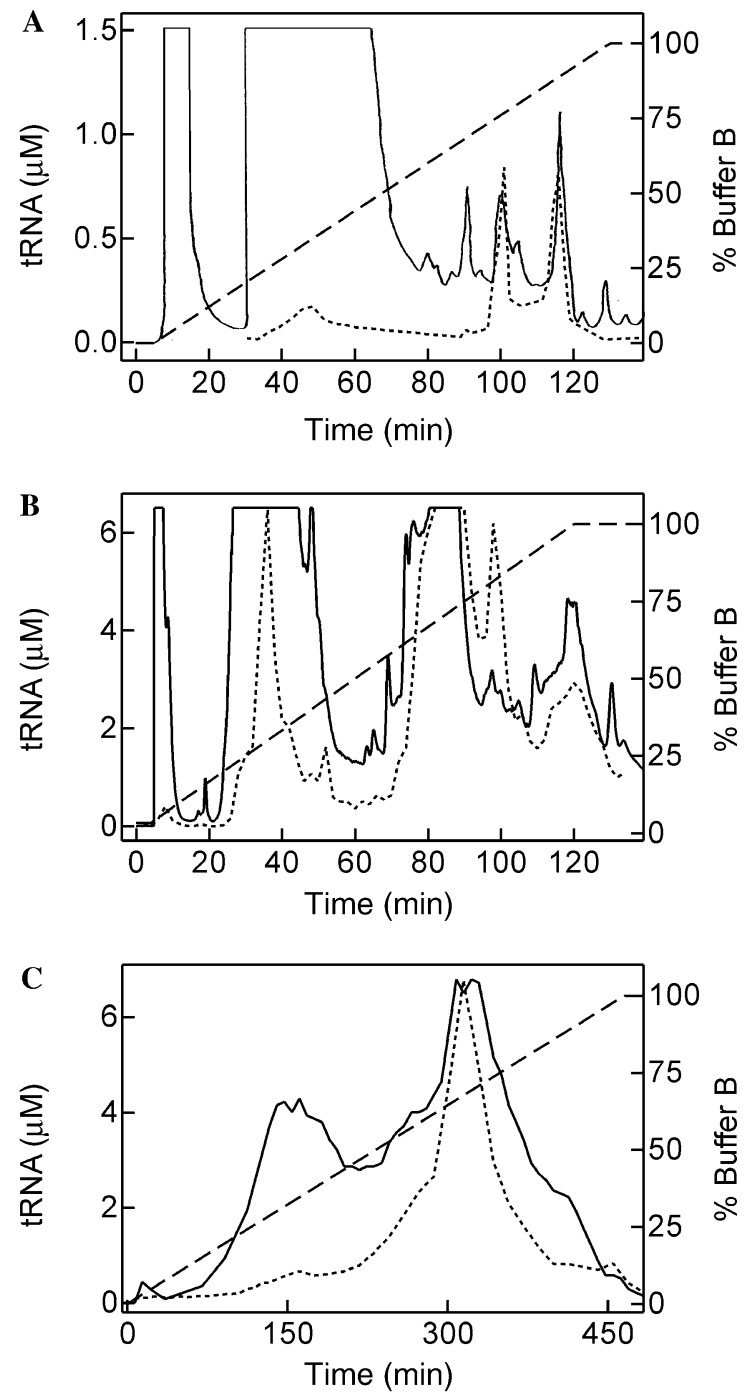

Fig. 2. Separation of Fmoc-aa-tRNA from total tRNA. Elution profiles were monitored by absorption at $260 \mathrm{~nm}(-)$ and ${ }^{14} \mathrm{C}$ radioactivity (--). The increase in buffer B percentage (--) is indicated. (A) Separation of Fmoc-Ala-tRNA ${ }^{\text {Ala }}$ from total tRNA by reversed-phase HPLC on a LiChrospher WP300 RP-18 $(5 \mu \mathrm{m})$ column $(250 \times 10 \mathrm{~mm})$ (Merck). $\left[{ }^{14} \mathrm{C}\right]$ Alanine $(50 \mathrm{dpm} / \mathrm{pmol})$ was detected by scintillation counting of $50 \mu$ l of each fraction $(4.5 \mathrm{ml})$ in Lumasafe Plus cocktail (Lumac*LSC). (B) Separation of Fmoc-Ser-tRNA ${ }^{\mathrm{Sec} / \mathrm{Ser}}$ from total tRNA (containing overexpressed tRNA $\left.{ }^{\mathrm{Sec}}\right)$ by reversed-phase HPLC. To measure $\left[{ }^{14} \mathrm{C}\right]$ serine $(5 \mathrm{dpm} / \mathrm{pmol}), 50 \mu \mathrm{l}$ of each fraction $(6 \mathrm{ml})$ was counted. (C) Separation of Fmoc-Ser-tRNA ${ }^{\text {Sec/Ser }}$ from total tRNA on Phenyl Sepharose $(75 \mathrm{ml})$. Here $100 \mu \mathrm{l}$ of each fraction $(10 \mathrm{ml})$ was counted to detect $\left[{ }^{14} \mathrm{C}\right]$ serine (6 dpm/pmol).

reaction. The second large peak at $20-50 \%$ buffer B contained total tRNA. The radioactivity profile indicated in this peak the presence of aa-tRNA that was not modified with FmocOSu. Finally, Fmoc-aa-tRNA eluted at 60 $100 \%$ buffer B. For either tRNA ${ }^{\text {Ala }}$ or tRNA ${ }^{\text {Sec/Ser }}$ preparations, two peaks that were well separated from bulk tRNA were found. For Fmoc-Ser-tRNA ${ }^{\mathrm{Sec} / \mathrm{Ser}}$, we could show (using analytical assays for the conversion of serine to selenocysteine) that the first of the two peaks contained tRNASec $\left(60-90 \%\right.$ buffer B), whereas tRNA ${ }^{\text {Ser }}$ isoacceptors eluted 
in the second peak (90-100\% buffer B) [10]. The two peaks of Fmoc-Ala-tRNA ${ }^{\text {Ala }}$ presumably contain the two isoacceptors of tRNA ${ }^{\mathrm{Ala}}$ [11]; the isoacceptor identity was not analyzed here. The modification efficiency of aa-tRNA with $\mathrm{FmocOSu}$ was approximately $70-80 \%$, as estimated from the ratio of ${ }^{14} \mathrm{C}$ radioactivity in the fractions containing aa-tRNA and Fmoc-aa-tRNA. The fractions containing Fmoc-aa-tRNA were pooled and precipitated with ethanol. As an alternative to reversed-phase HPLC, Phenyl Sepharose 6 Fast Flow (high sub) (GE Healthcare) can be used to separate the modified aa-tRNA from bulk tRNA (Fig. 2C) using a 1.4- to 0 -M gradient of ammonium sulfate in $50 \mathrm{mM}$ sodium acetate $(\mathrm{pH} 6.5)$ and $10 \mathrm{mM} \mathrm{MgCl}$.

To recover deacylated tRNA, Fmoc-aa-tRNA was deacylated by incubation with $1 \mathrm{M}$ Tris- $\mathrm{HCl}(\mathrm{pH}$ 9.0) at room temperature for $4 \mathrm{~h}$. This procedure resulted in more than $90 \%$ deacylation, as verified by analytical reversedphase HPLC under conditions similar to the preparative HPLC described above. Thus, efficient deacylation can be achieved without using expensive enzymes [12]. Deacylated tRNA was recovered by the addition of potassium acetate ( $\mathrm{pH} 4.5$ ) to $0.3 \mathrm{M}$ and ethanol precipitation. The purified tRNA $^{\text {Ala }}$ could be charged by alanine to $60 \%$, corresponding to approximately a 20 -fold enrichment in a single chromatographic step. The isolated $\mathrm{tRNA}^{\mathrm{Sec}}$, which was purified after overexpression in E. coli, also had a $60 \%$ acceptor activity.

The current method of tRNA purification has several advantages compared with previously published procedures $[9,12-14]$. It is generally applicable for all tRNAs without any adaptations in the described protocol because it relies solely on the selective aminoacylation by highly specific aa-tRNA synthetases and the subsequent modification of the amino group. In contrast to a previously published method [9], the ester used in the current work $(\mathrm{FmocOSu})$ is readily available at low cost and is stable. By our method, the tRNA can be rapidly enriched up to 20 -fold after only a single purification step. Both chromatographic materials (LiChrospher WP-300 RP-18 and Phenyl Sepharose) are more readily available than the BD cellulose that was used previously for tRNA purification by a similar approach [15]. Compared with other methods, such as affinity chromatography with immobilized elongation factor $\mathrm{Tu}$ (EF-Tu) [13] and streptavidin binding of N-biotinylated aa-tRNAs [14], the current method uses chromatographic materials that are significantly less expensive and allows purification in one chromatographic step of tRNAs in very large preparative amounts - up to $1700 A_{260}$ units on the LiChrospher WP300 RP-18 column or $4000 A_{260}$ units/200 ml Phenyl Sepharose. Thus, we have described a fast, inexpensive, and efficient method for the isolation of specific tRNAs of good purity. For more demanding tasks, such as the purification of individual tRNA isoacceptors, the described procedure represents an ideal first step that can be followed by further purification steps using HPLC or hydrophobic chromatography [3].

\section{Acknowledgments}

We thank V.I. Makhno for total tRNA preparation from E. coli MRE 600 and for technical advice; A. Bock for the plasmid pCB2013 used to overproduce tRNA ${ }^{\text {Sec}}$; and S. Moebitz, P. Striebeck, A. Boehm, and C. Schillings for expert technical assistance. This work was supported by the Deutsche Forschungsgemeinschaft, the Alfried Krupp von Bohlen und Halbach-Stiftung, and the Fonds der Chemischen Industrie. U. Kothe was supported by a fellowship of the Studienstiftung des deutschen Volkes.

\section{References}

[1] A.L. Konevega, N.G. Soboleva, V.I. Makhno, Y.P. Semenkov, W. Wintermeyer, M.V. Rodnina, V.I. Katunin, Purine bases at position 37 of tRNA stabilize codon-anticodon interaction in the ribosomal A site by stacking and $\mathrm{Mg}^{2+}$-dependent interactions, RNA 10 (2004) 90-101.

[2] P.F. Agris, Decoding the genome: a modified view, Nucleic Acids Res. 32 (2004) 223-238.

[3] N. Horie, Z. Yamaizumi, Y. Kuchino, K. Takai, E. Goldman, T. Miyazawa, S. Nishimura, S. Yokoyama, Modified nucleosides in the first positions of the anticodons of $\mathrm{tRNA}_{4}^{\mathrm{Leu}}$ and $\mathrm{tRNA}_{5}^{\mathrm{Leu}}$ from Escherichia coli, Biochemistry 38 (1999) 207-217.

[4] G. Ehrenstein, Isolation of sRNA from intact Escherichia coli cells, Methods Enzymol. 12 (1967) 588-596.

[5] M. Thanbichler, A. Bock, Selenoprotein biosynthesis: purification and assay of components involved in selenocysteine biosynthesis and insertion in Escherichia coli, Methods Enzymol. 347 (2002) 3-16.

[6] C. Baron, A. Bock, The length of the aminoacyl-acceptor stem of the selenocysteine-specific tRNA ${ }^{\mathrm{Sec}}$ of Escherichia coli is the determinant for binding to elongation factors SELB or Tu, J. Biol. Chem. 266 (1991) 20375-20379.

[7] K.S. Kemkhadze, V.B. Odintsov, Y.P. Semenkov, S.V. Kirillov, Quantitative study of the interaction of aminoacyl-tRNA with the A site of Escherichia coli ribosomes: Equilibrium and kinetic parameters of binding in the absence of EF-Tu factor and GTP, FEBS Lett. 125 (1981) 10-14.

[8] M.V. Rodnina, Y.P. Semenkov, W. Wintermeyer, Purification of fMet-tRNA $^{\text {fMet }}$ by fast protein liquid chromatography, Anal. Biochem. 219 (1994) 380-381.

[9] I. Gillam, D. Blew, R.C. Warrington, M. von Tigerstrom, G.M. Tener, A general procedure for the isolation of specific transfer ribonucleic acids, Biochemistry 7 (1968) 3459-3468.

[10] J.T. Kaiser, K. Gromadski, M. Rother, H. Engelhardt, M.V. Rodnina, M.C. Wahl, Structural and functional investigation of a putative archaeal selenocysteine synthase, Biochemistry 44 (2005) 13315-13327.

[11] H. Dong, L. Nilsson, C.G. Kurland, Co-variation of tRNA abundance and codon usage in Escherichia coli at different growth rates, J. Mol. Biol. 260 (1996) 649-663.

[12] E. Cayama, A. Yepez, F. Rotondo, E. Bandeira, A.C. Ferreras, F.J. Triana-Alonso, New chromatographic and biochemical strategies for quick preparative isolation of tRNA, Nucleic Acids Res. 28 (2000) e64.

[13] S. Ribeiro, S. Nock, M. Sprinzl, Purification of aminoacyl-tRNA by affinity chromatography on immobilized Thermus thermophilus EFTu · GTP, Anal. Biochem. 228 (1995) 330-335.

[14] J. Putz, J. Wientges, M. Sissler, R. Giege, C. Florentz, A. Schwienhorst, Rapid selection of aminoacyl-tRNAs based on biotinylation of $\alpha-\mathrm{NH}_{2}$ group of charged amino acids, Nucleic Acids Res. 25 (1997) 1862-1863.

[15] I. Gillam, S. Millward, D. Blew, M. von Tigerstrom, E. Wimmer, G.M. Tener, The separation of soluble ribonucleic acids on benzoylated diethylaminoethylcellulose, Biochemistry 6 (1967) 3043-3056. 